\title{
SÍNDROME DE RECONSTITUCIÓN INMUNE POR BCG EN NIÑOS TRATADOS POR VIH
}

\author{
Edwin Miranda-Choque ${ }^{1, a}$, Jorge Candela-Herrera', ${ }^{1, b}$, Eddy R. Segura ${ }^{2,3, c}$, \\ Sonia Farfán-Ramos ${ }^{4, \mathrm{~d}}$, Aldo Barriga ${ }^{1, \mathrm{e}}$
}

\section{RESUMEN}

El objetivo del estudio fue describir el perfil clínico del síndrome de reconstitución inmune por Mycobacterium bovis Bacillus Calmette-Guérin (SIRI-BCG) en niños con infección VIH que reciben tratamiento antirretroviral de gran actividad (TARGA) en el Instituto Nacional de Salud del Niño de Lima, Perú. Se realizó un estudio de serie de casos, que incluyó ocho niños con SIRI-BCG, definido como la presencia de linfadenopatía regional o inflamación en sitio de inoculación de BCG con disminución de al menos un logaritmo en la carga viral o mejoría inmunológica. Todos los pacientes tenían estadio SIDA (C3). La mediana de edad de inicio del TARGA fue de 7,2 meses y el evento se produjo entre 3 a 11 semanas luego de haberlo iniciado. En siete casos se produjo adenitis axilar. Al comparar con el grupo sin SIRI-BCG se encontró asociación significativa con la edad de inicio del TARGA de un año, estado de inmunodepresión severa, y carga viral incrementada. Se concluye que el SIRI-BCG está relacionado con una rápida progresión clínica de la infección VIH/SIDA de trasmisión vertical, estadio de inmunosupresión severa, y carga viral alta al momento del inicio del TARGA.

Palabras clave: VIH; BCG; Niño; Terapia antirretroviral altamente activa; Inmunología (fuente: DeCS BIREME).

\section{IMMUNE RECONSTITUTION SYNDROME DUE TO BCG IN HIV-TREATED CHILDREN}

\section{ABSTRACT}

The objective of this study is to describe the clinical profile of the immune reconstitution syndrome due to Mycobacterium bovis Bacillus Calmette-Guérin (IRS-BCG) in children with HIV infection who receive highly active antiretroviral treatment (HAART) at Instituto Nacional de Salud del Niño de Lima (National Children's Health Institute of Lima), Peru. A case study was conducted, including 8 children with IRS-BCG, defined as the presence of regional lymphadenopathy or inflammation on the BCG vaccination site with at least one less logarithm in the viral load or immune improvement. All patients had AIDS (C3). The starting median age in HAART was 7.2 months and the event occurred 3 to 11 weeks after the treatment was started. 7 cases showed axillary adenitis. When compared with the Non IRS-BCG group, a significant association between the age at which HAART was started at one year, severe immunodepression, and increased viral load was found. It is concluded that IRS-BCG was related to a rapid clinical progression of the motherto-child transmitted HIVIAIDS infection, severe immunosuppression and high viral load when the HAART began.

Key words: HIV; BCG; Child; Antiretroviral therapy, highly active; Immunology (source: MesH NLM).

\section{INTRODUCCIÓN}

La coinfección entre el complejo Mycobacterium tuberculosis y el virus de inmunodeficiencia humana (VIH) está relacionada con el incremento de la morbilidad y mortalidad, especialmente en países en vías de desarrollo como el Perú, por lo que la Organización Mundial de la Salud (OMS) recomienda la inmunización con la vacuna de BCG (M. bovis Bacillus Calmette-Guerín) a todos los recién nacidos, para evitar las complicaciones severas de tuberculosis (TB) en los primeros años de vida ${ }^{(1,2)}$.

\footnotetext{
Instituto Nacional de Salud del Niño. Lima, Perú.

Programa de Salud Global, Universidad de California-Los Ángeles. California, EE. UU.

Escuela de Posgrado "Víctor Alzamora Castro", Universidad Peruana Cayetano Heredia. Lima, Perú.

Hospital de Emergencias Pediátricas. Lima, Perú.

a Médico infectólogo; ${ }^{\mathrm{b}}$ médico pediatra; ${ }^{\mathrm{c}}$ médico magíster en Salud Pública y Epidemiología; ${ }^{\mathrm{d}}$ enfermera magíster en Salud Pública y Comunitaria, especialista en Enfermería Pediátrica; ${ }^{\mathrm{e}}$ médico cirujano

Recibido: 13-03-12 Aprobado: 14-11-12

* Este artículo se basa en el informe técnico "Síndrome de reconstitución inmune por Mycobacterium bovis Bacillus Calmette-Guérin en niños peruanos con tratamiento antirretroviral por HIV" disponible en la página web del Instituto Nacional de Salud del Niño de Lima, Perú.
}

Citar como: Miranda-Choque E, Candela-Herrera J, Segura ER, Farfán-Ramos S, Barriga A. Síndrome de reconstitución inmune por BCG en niños tratados por VIH. Rev Peru Med Exp Salud Publica. 2012;29(4):498-502. 
La vacuna BCG se prepara con bacilos vivos atenuados del Mycobacterium bovis y es considerada segura. Sin embargo, puede ocasionar reacciones adversas como linfadenitis supurativa, entre los dos a seis meses posterior a su inoculación, con una tasa de 100 a 1000 por 1 millón de dosis en pacientes sin $\mathrm{VIH}{ }^{(3)}$. La linfadenitis supurativa es una enfermedad regional inducida por la BCG la cual puede volverse una enfermedad diseminada. El síndrome inflamatorio de reconstitución inmune (SIRI) es un desorden inmunológico observado comúnmente en pacientes que inician TARGA, y que se relaciona con manifestaciones inflamatorias de intensidad variable como linfadenitis supurativa por mejora en la inmunidad, por tratamiento en pacientes que previamente son inmunológicamente deprimidos (1). En este caso si el agente es la BCG, se llama síndrome de reconstitución inmune por Mycobacterium bovis Bacillus Calmette-Guérin (SIRI-BCG).

Aunque no está indicado vacunar con BCG a niños infectados con VIH, por la posibilidad de desarrollar enfermedad diseminada por BCG el diagnóstico de VIH del niño o de la madre no siempre es oportuno, por lo que ocasionalmente se producen vacunaciones accidentales. Dado que en el Instituto Nacional de Salud del Niño (INSN) brinda atenciones al mayor número de niños infectados con $\mathrm{VIH}$ en el Perú, como parte de un programa de prevención y control de VIH/SIDA que incluye estrategias de prevención de la transmisión vertical y tratamiento antirretroviral de gran actividad (TARGA) ${ }^{(2)}$, y dado que no hemos encontrado estudios previos sobre el SIRI-BCG en el Perú; el objetivo del presente estudio tuvo como objetivo determinar el perfil clínico del SIRI-BCG en una serie de niños infectados con VIH en el INSN del 2007 al 2009.

\section{EL ESTUDIO}

El presente trabajo es un estudio tipo serie de casos de pacientes con $\mathrm{VIH}$ que presentaron SIRI-BCG. EI universo de pacientes incluye a los menores de 18 años de edad con diagnóstico de VIH confirmado por Western blot, PCR ADN VIH-1 y ELISA (detección de anticuerpos totales), que iniciaron TARGA entre 2007 y 2009 y que recibieron vacunación con BCG en el INSN.

De estos, se incluyó aquellos que presentaron una linfoadenopatía regional ipsilateral o signos inflamatorios en la región de inoculación de la vacuna con presencia de BAAR (bacilo ácido alcohol resistente) en la baciloscopía o cultivo Löwenstein Jensen. Se excluyó a los menores que abandonaron el TARGA o a quienes no tenían información completa en sus historias clínicas.
De las historias clínicas se obtuvo información sobre conteo de linfocitos CD4, carga viral, fecha del evento SIRI$B C G$, esquema TARGA, sintomatología, características microbiológicas y seguimiento hasta un año.

Se incluyó además, los datos de citometría de flujo de CD4 realizados en los laboratorios del Instituto Nacional de Salud (INS) de Perú. Se calculó el porcentaje de CD4 como valor absoluto dividido por el valor de linfocitos totales de sangre periférica por $\mathrm{mL}$. Este valor se utilizó para la evaluación del estado inmunológico según las recomendaciones de la OMS y el Centro para el Control y la Prevención de Enfermedades (CDC), de la siguiente manera: inmunosupresión severa para menores a 11 meses si $<25 \%$ o $<1500 \mathrm{cel} / \mathrm{mm}$; para 12 meses a 35 meses si $<20 \%$ o $<750 \mathrm{cel} / \mathrm{mm}$; para 36 a 59 meses si $<15 \%$ o $<350 \mathrm{cel} / \mathrm{mm}$; para mayores a 5 años si $<15 \%$ o $<200 \mathrm{cel} / \mathrm{mm}^{(2,3)}$. La carga viral (CV) fue detectada hasta límites bajos de 40 copias $/ \mathrm{mL}$. El recuentro de CD4 y CV se realizó en dos momentos: al inicio y a los seis meses de iniciado el TARGA.

El estado nutricional de los participantes fue evaluado mediante el programa WHO Anthro (versión 3.2.2, Geneva, Switzerland) y las tablas de la OMS, clasificándolos como retardo del crecimiento (talla para la edad $z$ score $\leq 2$ SD debajo del promedio), emaciación (peso para la talla $z$ score $\leq 2$ SD debajo del promedio), bajo peso (peso para la edad $z$ score $\leq 2$ SD debajo del promedio) y alteración de la nutrición (si presenta al menos uno de los tres parámetros mencionados anteriormente).

A la actualidad no hay pruebas confirmatorias de SIRI. El diagnóstico se realizó mediante criterios clínicos y de laboratorio, que incluyen: 1) evidencia de mejoría al TARGA mediante la disminución en plasma de la carga viral (VIH ARN > 1 log10 o CV < 400 copias $/ \mathrm{mL}$ en el control) ${ }^{(4)}$; y 2) deterioro clínico por una infección o inflamación condicionada al inicio del TARGA. La mejoría en la respuesta inmunológica fue definida como un incremento de la línea de base del conteo de células CD4 de más de $5 \%$ o un total del conteo de células CD4 de más de 250 células/mL ${ }^{(1,5,6)}$.

Se realizó un análisis descriptivo de las características clínicas de los pacientes. De forma secundaria, se evaluaron diferencias entre los que presentaron SIRIBCG con la población universo (pacientes SIBI-BCG negativos) según características demográficas y clínicas mediante la prueba de chi cuadrado o la prueba exacta de Fisher para variables categóricas y la prueba de $U$ Mann-Whitney para las cuantitativas. Se consideró una diferencia significativa valor de $p<0,05$. Se utilizó el programa STATA versión 10. 
El estudio fue aprobado por el comité de ética del Instituto Nacional de Salud del Niño con código CL-692010.

\section{HALLAZGOS}

Entre los años 2007 y 2009, cien niños iniciaron TARGA en el INSN; se excluyó a 17 pacientes (seis por abandono al TARGA o por ser transferidos a otro hospital y once por datos incompletos en la historia clínica). De los 83 pacientes que podrían ser considerados, se encontró ocho casos de SIRI-BCG (9,6\%). De estos, cuatro fueron varones; el evento se produjo entre tres a once semanas luego de haber iniciado el TARGA (mediana de 29 días; RIQ: 27,0 - 50,5 días). La mediana de edad de inicio del TARGA fue de 7,2 meses (RIQ: 6 - 9 meses) (Tabla 1).

Los ocho casos con el evento fueron negativos a la prueba de tuberculina antes del inicio del TARGA. Posteriormente, reaccionaron tres pacientes con diámetros de 9 a $12 \mathrm{~cm}$. Siete casos SIRI-BCG tuvieron diagnóstico de adenitis regional y un caso desarrolló absceso en hombro derecho, todos con confirmación por baciloscopía.

Todos los casos fueron detectados posnatalmente infectados con VIH en estadios sintomáticos severos;

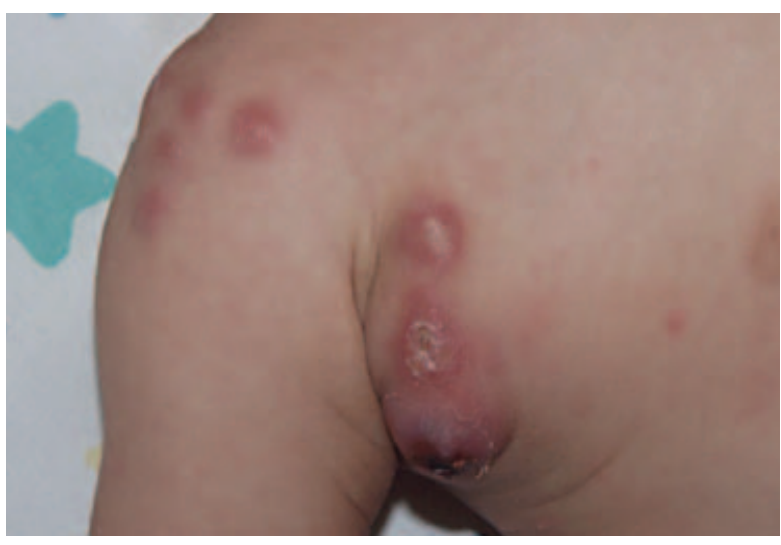

Figura 1. Paciente de nueve meses de edad con diagnóstico de linfadenitis por SIRI-BCG. El evento se produjo 30 días después de iniciar TARGA.

seis pacientes recibieron tratamiento antimicobacteriano; de ellos, dos recibieron pirazinamida en el esquema de tratamiento. Ningún paciente falleció.

Se exploró la asociación entre los casos de SIRI-BCG con el grupo que no lo desarrollaron. De los 83 niños incluidos (ocho con SIRI-BCG), el 45,8\% (38) fueron varones y el promedio de edad de inicio de TARGA fue 3,4 años (rango: 3 meses a 17 años). La vía más

Tabla 1. Características de la enfermedad y el tratamiento de los casos de SIRI-BCG, en el INSN, 2007-2009.

\begin{tabular}{|c|c|c|c|c|c|c|c|}
\hline Caso & $\begin{array}{l}\text { Edad al } \\
\text { diagnóstico } \\
\text { SIRI-BCG }\end{array}$ & $\begin{array}{l}\text { Días en TARGA } \\
\text { al diagnóstico } \\
\text { SIRI-BCG }\end{array}$ & $\begin{array}{l}\text { Esquema } \\
\text { TARGA }\end{array}$ & $\begin{array}{l}\text { Estadio } \\
\text { VIH }\end{array}$ & Evidencia clínica & $\begin{array}{l}\text { Característica } \\
\text { microbiológica }\end{array}$ & Tratamiento \\
\hline 1 & 9 meses & 30 días & d4t 3tc nfv & C3 & $\begin{array}{l}\text { Fiebre, nódulo axilar derecho au- } \\
\text { mentado de tamaño de } 4,5 \times 5 \mathrm{~cm} \\
\text { diámetro }\end{array}$ & $\begin{array}{l}\text { Aspirado absceso } \\
\text { BAAR ++ cultivo } 2 \\
\text { colonias }\end{array}$ & HES Z \\
\hline 2 & 1 año 1 mes & 28 días & AZT 3tc nfv & C3 & $\begin{array}{l}\text { Fiebre, nódulo axilar derecho au- } \\
\text { mentado de tamaño, blando, móvil } \\
5 \mathrm{~cm} \text { diámetro, evolución en } 10 \text { días }\end{array}$ & $\begin{array}{l}\text { Biopsia BAAR + } \\
\text { cultivo negativo }\end{array}$ & H R FQ S (Z) \\
\hline 3 & 6 meses & 75 días & AZT 3tc lop-r & C3 & $\begin{array}{l}\text { Masa hombro derecho en } 2 \text { semanas } \\
\text { crece de } 1 \text { a } 2,5 \mathrm{~cm} \text {, absceda y fistu- } \\
\text { liza posteriormente }\end{array}$ & $\begin{array}{l}\text { Aspirado absceso } \\
\text { BAAR }+ \text { cultivo } \\
\text { negativo }\end{array}$ & $\begin{array}{l}\text { No tratamiento } \\
\text { especifico }\end{array}$ \\
\hline 4 & 4 meses & 21 días & d4t 3tc nfv & C3 & $\begin{array}{l}\text { Fiebre, nódulo axilar derecho aumen- } \\
\text { tado de tamaño de } 5 \times 5 \mathrm{~cm} \text { de diá- } \\
\text { metro, crecimiento en } 5 \text { días, flogosis }\end{array}$ & $\begin{array}{c}\text { Aspirado directo } \\
\text { BAAR }++, \text { cultivo } \\
\text { negativo }\end{array}$ & HE S FQ \\
\hline 5 & 5 meses & 53 días & AZT 3tc lop-r & C3 & $\begin{array}{l}\text { Adenopatía axilar derecho bordes } \\
\text { regulares no doloroso, flogosis y } \\
\text { fistuliza posteriormente, en zona de } \\
\text { brazo derecho (sitio de vacuna) }\end{array}$ & $\begin{array}{l}\text { Aspirado absceso } \\
\text { BAAR + cultivo } \\
\text { negativo }\end{array}$ & $\begin{array}{c}\text { No tratamiento } \\
\text { especifico }\end{array}$ \\
\hline 6 & 7 meses & 28 días & AZT 3tc nfv & C3 & $\begin{array}{l}\text { Fiebre, nódulo axilar derecho aumenta- } \\
\text { do de tamaño } 4 \times 4,5 \mathrm{~cm} \text {, crece en } 3 \text { días }\end{array}$ & $\begin{array}{l}\text { Aspirado absceso } \\
\text { BAAR }+ \text { cultivo } 2 \\
\text { colonias }\end{array}$ & HRE \\
\hline 7 & 6 meses & 48 días & d4t 3tc Lop-r & C3 & $\begin{array}{l}\text { Nódulo axilar derecho aumentado de } \\
\text { tamaño, blando móvil } 1,5 \mathrm{~cm} \text { diáme- } \\
\text { tro, evolución en } 10 \text { días }\end{array}$ & $\begin{array}{c}\text { Aspirado Absceso } \\
\text { BAAR +++ cultivo } \\
\text { negativo }\end{array}$ & HES \\
\hline 8 & 1 año 4 meses & 26 días & d4t 3tc Lop-r & C3 & $\begin{array}{l}\text { Nódulo axilar derecho aumentado de } \\
\text { tamaño, blando móvil } 4 \mathrm{~cm} \text { diámetro, } \\
\text { evolución en } 5 \text { días }\end{array}$ & $\begin{array}{l}\text { Aspirado Absceso } \\
\text { BAAR } 8 \text { cultivo } \\
\text { negativo }\end{array}$ & E FQ S \\
\hline
\end{tabular}

BAAR: Bacilo ácido alcohol resistente; $\mathrm{H}$ : isoniazida; Z: pirazinamida; R: rifampizina; FQ: fluoroquinolona; S: estreptomicina; C3: estadio clínico C3 o SIDA; d4t: estavudina; AZT: zidovudina; 3tc: lamivudina; nfv: nelfinavir; Lop-r: lopinavir-ritonavir.

Datos adicionales están disponibles como Anexos en www.rpmesp.ins.gob.pe 
frecuente de transmisión del VIH fue la vertical (75/83). El esquema TARGA de inicio que incluía zidovudina (o estavudina), lamivudina y nelfinavir se aplicó en 35 pacientes, esquemas con zidovudina (o estavudina), lamivudina y nevirapina en 18 , esquemas con zidovudina (o estavudina), lamivudina y efavirenz en 17, esquemas con zidovudina (o estavudina), lamivudina y lopinavir/rit en 12. Solo un paciente recibió lopinavir/rit, atazanavir y lamivudina. La categoría de inmunosupresión severa (según la definición OMS) se encontró en el 62,7\% de los casos. Las características epidemiológicas, clínicas y de laboratorio se encuentran en la Tabla 1.

Se encontró una diferencia significativa con la edad de inicio menor de un año $(p=0,032)$, estado de inmunosupresión severa $(p=0,03)$ y la magnitud de la carga viral $(p=0,001)$ siendo marginal la asociación con algún grado de desnutrición ( $p=0,083)$ (Tabla 2).

\section{DISCUSIÓN}

El presente estudio es la serie nacional más amplia de casos SIRI-BCG en niños con VIH. El perfil clínico encontrado es de un niño menor de 2 años con VIH, infectado verticalmente, con inicio reciente de TARGA, estadio de inmunosupresión severa y con carga viral mayor a $5 \log ^{10}$ de copias $/ \mathrm{mm}^{3}$, datos similares a lo encontrado en la literatura internacional ${ }^{(7-9)}$.

Cabe señalar que los casos de SIRI-BCG en la presente investigación se debieron a vacunaciones accidentales al desconocerse la infección $\mathrm{VIH}$ de la madre o el recién nacido. Diversos reportes de casos de SIRI-BCG de países en vías de desarrollo ${ }^{(10,11)}$ ponen en debate las recomendaciones de la OMS de evitar la vacunación en niños con VIH ${ }^{(12-14)}$. En el Perú, considerando que la transmisión vertical es menor al $2 \%$ en la población de madres que recibieron las medidas adecuadas de prevención vertical, creemos que no debería contraindicarse la vacunación con BCG a los recién nacidos asintomáticos de madres con infección $\mathrm{VIH}$ captados por el sistema de salud ${ }^{(15)}$.

En el caso se demuestre la condición de infección en el niño por la prueba de PCR VIH entre las 4 y 8 semanas de edad, estaría indicado iniciar inmediatamente el TARGA de acuerdo a lo normado por el MINSA. Así se obtendría beneficios a los recién nacidos expuestos no infectados con VIH (asumiendo que sería el $98 \%$ ) en la prevención de la infección por TB y disminuir el riesgo de desarrollar formas severas de la enfermedad.

Si consideramos que el MINSAestimó que en el año 2009, 835 madres infectadas con VIH requerían TARGA y solo $560(67,0 \%)$ lo recibieron, hace suponer que el grupo restante (sin TARGA) tendrían niños infectados con VIH y vacunados con BCG, diagnosticados probablemente en estadios sintomáticos de la enfermedad, posterior al periodo neonatal ${ }^{(15)}$.

El presente estudio presenta la limitación de no contar con la confirmación por cultivo para micobacterias del $M$. bovis Bacillus Calmette-Guérin. Asimismo, si bien el INSN es un hospital de referencia nacional, no representa la totalidad de casos. Por ello, la información secundaria, tal como las asociaciones, debe ser considerada con prudencia.

En conclusión, el SIRI-BCG está relacionado a una rápida progresión clínica de la infección VIH/SIDA de

Tabla 2. Características demográficas y de laboratorio de niños que iniciaron TARGA en el INSN, periodo $2007-2009$.

\begin{tabular}{|c|c|c|c|}
\hline Característica & $\begin{array}{c}\text { BCG-SIRI } \\
\left(\mathrm{N} .^{\circ}=8\right)\end{array}$ & $\begin{array}{c}\text { No BCG-SIRI } \\
\left(\mathrm{N} .^{\circ}=75\right)\end{array}$ & $p$ \\
\hline \multicolumn{4}{|l|}{ Edad inicio TARGA } \\
\hline Mediana (RIQ) & $0,6(0,5-0,9)$ & $2(0,8-5,0)$ & 0,013 \\
\hline \multicolumn{4}{|l|}{ Sexo } \\
\hline Hombres & $4(50,0 \%)$ & $34(45,4 \%)$ & 0,801 \\
\hline Mujeres & $4(50,0 \%)$ & $41(54,6 \%)$ & \\
\hline \multicolumn{4}{|l|}{ Estadio inmunológico } \\
\hline Inmunosupresión severa & $8(100,0 \%)$ & $47(62,7 \%)$ & 0,030 \\
\hline No inmunosupresión severa & 0 & 28 & \\
\hline \multicolumn{4}{|l|}{ Carga viral } \\
\hline Mediana & 2713756 & 131325 & 0,001 \\
\hline $\mathrm{RIQ}$ & $704123-9352814$ & $76000-341875$ & \\
\hline \multicolumn{4}{|l|}{ Algún indicador de malnutrición } \\
\hline Según peso y talla & $8(100,0 \%)$ & $54(72,0 \%)$ & 0,083 \\
\hline No malnutrición & 0 & 21 & \\
\hline
\end{tabular}

BCG-SIRI: síndrome inflamatorio de reconstitución inmune a la vacuna de la BCG; RIQ: rango intercuartil 
transmisión vertical y estadio de inmunosupresión severa al momento del inicio del TARGA. La incidencia es baja en los pacientes con VIH que recibieron TARGA tras ser vacunados con $B C G$.

Agradecimientos: al personal del Servicio de Infectología del INSN.

Descargo de Responsabilidad: las opiniones y afirmaciones contenidas aquí son propias de los autores y no deben interpretarse como posición oficial del INSN.
Contribuciones de autoría: $\mathrm{EMCH}$ y $\mathrm{AB}$ participaron en la concepción y diseño del estudio. EMCH, JCH, SFR y ESR en la interpretación de los resultados. ESR en el análisis estadístico. $\mathrm{EMCH}, \mathrm{AB}, \mathrm{SFR}$ y $\mathrm{JCH}$ en la redacción de resultados y del manuscrito. Todos los autores dieron su aprobación final del manuscrito.

Fuentes de financiamiento: autofinanciado.

Conflictos de interés. Los autores declaran no tener conflictos de interés en la publicación de este artículo.

\section{REFERENCIAS BIBLIOGRÁFICAS}

1. Centers for Disease Control (CDC). BCG vaccination and pediatric HIV infection--Rwanda, 1988-1990. MMWR Morb Mortal Wkly Rep. 1991;40(48):833-6.

2. HIV patients can be vaccinated against TB. Results show a whole cell vaccine works. AIDS Alert. 2010;25(7):78-9.

3. Organización Panamericana de Salud. Vacunación segura: módulos de capacitación. Módulo I Autoridad Regulatoria Nacional. Washington, DC: OPS; 2007.

4. Hesseling AC, Marais BJ, Gie RP, Schaaf HS, Fine PE, Godfrey-Faussett $\mathrm{P}$, et al. The risk of disseminated BacilleCalmette-Guerin (BCG) disease in HIV-infected children. Vaccine. 2007;25(1):14-8.

5. Nuttall JJ, Eley BS. BCG Vaccination in HIV-Infected Children. Tuberc Res Treat. 2011;2011:712736.

6. Organización Mundial de la Salud (OMS) (Internet). Organización Mundial de la Salud. Inmunización Perfiles-Perú. [citado el 27 de enero del 2011]. disponible en: http://apps.who. int/inmunization_monitoring/.

7. Koppel A, Leonardo-Guerrero J, Rives S, Paniagua-Torres N, Sparrow C, Beck-Sagué CM. Immune reconstitution inflammatory syndrome due to Mycobacterium bovis Bacillus
Calmette-Guerin in infants receiving highly active antiretroviral therapy: a call for universal perinatal rapid HIV testing prior to administration of BCG immunization of neonates. J Trop Pediatr. 2010;56(4):280-3.

8. Hesseling AC, Caldwell J, Cotton MF, Eley BS, Jaspan HB, Jennings $\mathrm{K}$, et al. BCG vaccination in South African HIV-exposed infants--risks and benefits. S Afr Med J. 2009;99(2):88-91.

9. De Souza Campos Fernandes RC, Medina-Acosta E. BCG-itis in two antiretroviral-treated HIVinfected infants. Int J STD AIDS. 2010;21(9):662-3.

10. Perú, Ministerio de Salud. Norma técnica para el tratamiento antiretroviral de gran actividad TARGA en adultos infectados por el virus de la inmunodeficiencia humana. NT No 004-MINSA/DGSP-V.02. Lima: MINSA; 2005.

11. Perú, Ministerio de Salud, Dirección General de Epidemiologia. Perú: Informe nacional sobre los progresos realizado en la aplicación del UNGASS. Periodo: enero 2008 - diciembre 2010. Lima: MINSA; 2010.

12. Boulware DR, Callens S, Pahwa S. Pediatric HIV immune reconstitution inflammatory syndrome. Curr Opin HIV AIDS. 2008;3(4):461-7.
13. Schneider E, Whitmore S, Glynn KM, Dominguez K, Mitsch A, McKenna MT, et al. Revised surveillance case definitions for HIV infection among adults, adolescents, and children aged $<18$ months and for HIV infection and AIDS among children aged 18 months to $<13$ years--United States, 2008. MMWR Recomm Rep. 2008;57(RR10):1-12.

14. Murdoch DM, Venter WD, Van Rie A, Feldman C. Immune reconstitution inflammatory syndrome (IRIS): review of common infectious manifestations and treatment options. AIDS Res Ther. 2007;4:9.

15. Perú, Ministerio de Salud, Dirección General de Epidemiologia. Situación del VIH/Sida en el Perú. Boletín Epidemiológico Mensual [Internet]. 2010;12 [citado el 17 de mayo del 2011]. Disponible en: http://www.dge. gob.pe/vigilancia/vih/Boletin_2010/ diciembre.pdf

Correspondencia: Edwin Miranda Choque Dirección: Pachacutec 910, Lima 11, Perú. Teléfono: (51) 994645290

Correo electrónico: Emirandach@hotmail. com. 